\title{
A note on the uniqueness of weak solutions for the Navier-Stokes equations
}

\author{
Sadek Gala
}

Communicated by Jerry Bona, received December 13, 2008.

\begin{abstract}
Consider the Navier-Stokes equations with the initial data $a \in$ $L_{\sigma}^{2}\left(\mathbb{R}^{d}\right)$. Let $u$ and $v$ be two weak solutions with the same initial value $a$. If $\nabla u \in L^{\frac{2}{2-r}}\left((0, T) ; \dot{X}_{r}\left(\mathbb{R}^{d}\right)^{d}\right)$ where $\dot{X}_{r}\left(\mathbb{R}^{d}\right)$ is the multiplier space (see the definition in the text), then we have $u=v$.
\end{abstract}

\section{Contents}

1. Introduction 385

2. Uniqueness theorem 386

$\begin{array}{ll}\text { References } & 391\end{array}$

\section{Introduction}

Consider the Navier-Stokes equations in $(0, T) \times \mathbb{R}^{d}$ with $0<T<\infty$ and $d \geq 3$

$$
\begin{aligned}
\partial_{t} u+(u . \nabla) u-\Delta u+\nabla p & =0, & & (x, t) \in \mathbb{R}^{d} \times(0, \infty), \\
\nabla . u & =0, & & (x, t) \in \mathbb{R}^{d} \times(0, \infty), \\
u(x, 0) & =a(x), & & x \in \mathbb{R}^{d},
\end{aligned}
$$

where $u=u(x, t)$ is the velocity field, $p=p(x, t)$ is the scalar pressure and $a(x)$ with $\operatorname{div} a=0$ in the sense of distribution is the initial velocity field. For simplicity, we assume that the external force has a scalar potential and is included into the pressure gradient.

In their famous paper, Leray $[\mathbf{8}]$ and Hopf $[\mathbf{3}]$ constructed a weak solution $u$ of (1.1) for arbitrary $a \in L_{\sigma}^{2}$. The solution is called the Leray-Hopf weak solution. In the general case the problem on uniqueness of Leray-Hopf's weak solutions is still open question. Masuda [9] extended Serrin's class for uniqueness of weak solutions

1991 Mathematics Subject Classification. 35, 76.

Key words and phrases. Navier-Stokes equations, weak solution, multiplier space. 
and made it clear that the class $L^{\infty}\left((0, T) ; L^{d}\left(\mathbb{R}^{d}\right)\right)$ plays an important role for uniqueness of weak solutions. Kozono-Sohr [5] showed that the uniqueness holds in $L^{\infty}\left((0, T) ; L^{d}\right)$.

Foias [1] and Serrin [10] introduced the class $L^{\alpha}\left((0, \infty) ; L^{q}\right)$ and showed that under the additional assumption

$$
u \in L^{\alpha}\left((0, \infty) ; L^{q}\right) \quad \text { for } \quad \frac{2}{\alpha}+\frac{d}{q}=1 \quad \text { with } q>d,
$$

$u$ is the only weak solution.

The purpose of this note is to improve the criterion on uniqueness of weak solutions to in the class $L^{\frac{2}{2-r}}\left((0, T) ; \dot{X}_{r}\left(\mathbb{R}^{d}\right)^{d}\right)$. We know that for every $a \in L_{\sigma}^{2}\left(\mathbb{R}^{d}\right)$, there is at least one weak solution $u$ of (1.1) satisfying the energy inequality. Here we mean by the weak solution a function $u$ in $u \in L^{\infty}\left((0, T) ; L_{\sigma}^{2}\right) \cap L^{2}\left((0, T) ; \dot{H}_{\sigma}{ }^{1}\right)$ satisfying (1.1) in the sense of distributions (Definition 2). For more facts concerning uniqueness of weak solutions, we refer to a celebrated paper of Kozono and Sohr [5] (see also [2]).

Now, we give a description of the multiplier space $\dot{X}_{r}$ introduced recently by P.G. Lemarié-Rieusset in his work [6] (see also [7]). The space $\dot{X}_{r}$ of pointwise multipliers which map $L^{2}$ into $\dot{H}^{-r}$ is defined in the following way

Definition 1. For $0 \leq r<\frac{d}{2}$, we define the homogeneous space $\dot{X}_{r}$ by

$$
\dot{X}_{r}=\left\{f \in L_{l o c}^{2}: \forall g \in \dot{H}^{r} \quad f g \in L^{2}\right\}
$$

where we denote by $\dot{H}^{r}\left(\mathbb{R}^{d}\right)$ the completion of the space $\mathcal{D}\left(\mathbb{R}^{d}\right)$ with respect to the norm $\|u\|_{H^{r}}=\left\|(-\Delta)^{\frac{r}{2}} u\right\|_{L^{2}}$.

The norm of $\dot{X}_{r}$ is given by the operator norm of pointwise multiplication

$$
\|f\|_{\dot{X}_{r}}=\sup _{\|g\|_{H^{r}} \leq 1}\|f g\|_{L^{2}}
$$

We have the homogeneity properties : $\forall x_{0} \in \mathbb{R}^{d}$

$$
\begin{aligned}
\left\|f\left(.+x_{0}\right)\right\|_{\dot{X}_{r}} & =\|f\|_{\dot{X}_{r}} \\
\|f(\lambda .)\|_{\dot{X}_{r}} & =\frac{1}{\lambda^{r}}\|f\|_{\dot{X}_{r}}, \quad \lambda>0 .
\end{aligned}
$$

Additionally, for $0 \leq r<\frac{d}{2}$, we have the following inclusion relations :

$$
L^{\frac{d}{r}}\left(\mathbb{R}^{d}\right) \subset L^{\frac{d}{r}, \infty}\left(\mathbb{R}^{d}\right) \subset \dot{X}_{r}\left(\mathbb{R}^{d}\right) .
$$

where $L^{p, \infty}$ denotes the usual Lorentz (weak $L^{p}$ ) space. For the definition and basic properties of Lorentz spaces $L^{p, q}$ we refer to [11].

\section{Uniqueness theorem}

Before turning our attention to uniqueness issues, we start with some prerequisites for our main result. Let

$$
C_{0, \sigma}^{\infty}\left(\mathbb{R}^{d}\right)=\left\{\varphi \in\left(C_{0}^{\infty}\left(\mathbb{R}^{d}\right)\right)^{d}: \operatorname{div} \varphi=0\right\} \subseteq\left(C_{0}^{\infty}\left(\mathbb{R}^{d}\right)\right)^{d} .
$$


The subspace

$$
L_{\sigma}^{2}\left(\mathbb{R}^{d}\right)=\overline{C_{0, \sigma}^{\infty}\left(\mathbb{R}^{d}\right)}\|\cdot\|_{L^{2}}=\left\{u \in L^{2}\left(\mathbb{R}^{d}\right)^{d}: \operatorname{div} u=0\right\}
$$

obtained as the closure of $C_{0, \sigma}^{\infty}$ with respect to $L^{2}$-norm $\|\cdot\|_{L^{2}}$. $H_{\sigma}^{r}$ denotes the closure of $C_{0, \sigma}^{\infty}$ with respect to the norm

$$
\|u\|_{H^{r}}=\|u\|_{L^{2}}+\left\|(1-\Delta)^{\frac{r}{2}} u\right\|_{L^{2}}, \text { for } r \geq 0 .
$$

Our definition of Leray-Hopf weak solutions (see e.g. [5]) now reads :

Definition 2 (weak solutions). Let $a \in L_{\sigma}^{2}$ and $T>0$. A measurable function $u$ is called a weak solution of (1.1) on $(0, T)$ if $u$ satisfies the following properties

(1): $u \in L^{\infty}\left((0, T) ; L_{\sigma}^{2}\right) \cap L^{2}\left((0, T) ; \dot{H}_{\sigma}^{1}\right)$ for all $T>0$;

(2): $u(t)$ is continuous in time in the weak topology of $L_{\sigma}^{2}$ with

$$
\langle u(t), \phi\rangle \rightarrow\langle a, \phi\rangle \quad \text { as } t \rightarrow 0^{+}
$$

for all $\phi \in L_{\sigma}^{2}$;

(3): for any $0 \leq s \leq t \leq T$, u satisfies the identity

$$
\int_{s}^{t}\left\{-\left\langle u, \partial_{\tau} \phi\right\rangle+\langle u . \nabla u, \phi\rangle+\langle\nabla u, \nabla \phi\rangle\right\} d \tau=-\langle u(t), \phi(t)\rangle+\langle u(s), \phi(s)\rangle
$$

for all $\phi \in H^{1}\left((s, t) ; H_{\sigma}^{1}\right)$. Here $\langle.,$.$\rangle denotes the scalar product and \|\cdot\|_{L^{2}}$ denotes the norm in $L^{2}\left(\mathbb{R}^{d}\right)^{d}$.

REMARK 1. For $u$ and $\phi$ as above, the integral

$$
\int_{0}^{T}\langle u . \nabla u, \phi\rangle d \tau
$$

is well defined since we have by the Sobolev inequality

$$
\|u\|_{L^{\frac{2 d}{d-2}}} \leq C\|\nabla u\|_{L^{2}}
$$

that

$$
\begin{aligned}
\left|\int_{0}^{T}\langle u \cdot \nabla u, \phi\rangle d \tau\right| & \leq \int_{0}^{T}\|u\|_{L^{\frac{2 d}{d-2}}}\|\nabla u\|_{L^{2}}\|\phi\|_{L^{d}} d \tau \\
& \leq C \sup _{0<t<T}\|\phi\|_{L^{d}} \int_{0}^{T}\|\nabla u\|_{L^{2}}^{2} d \tau
\end{aligned}
$$

Existence of weak solutions has been established by Leray in [8] for initial velocity in $L_{\sigma}^{2}\left(\mathbb{R}^{d}\right)$. The result is the following

TheOREM 1 (Leray - Hopf). Let $T>0$. Then, for any given $a \in L_{\sigma}^{2}\left(\mathbb{R}^{d}\right)$, there exits at least one weak solution $u$ to (1.1) on $(0, T)$ such that

$$
\|u(t)\|_{L^{2}}^{2}+2 \int_{0}^{t}\|\nabla u(s)\|_{L^{2}}^{2} d s \leq\|a\|_{L^{2}}^{2}, \quad 0 \leq t<T .
$$

and

$$
\|u(t)-a\|_{L^{2}} \rightarrow 0 \quad \text { as } t \rightarrow+0
$$


Let us introduced the class $L^{s}\left((0, T) ; L^{\gamma}\right)$ with the norm $\|\cdot\|_{L^{s}\left((0, T) ; L^{\gamma}\right)}$

$$
\|u\|_{L^{s}\left((0, T) ; L^{\gamma}\right)}=\left(\int_{0}^{T}\|u(t)\|_{L^{\gamma}}^{s} d t\right)^{\frac{1}{s}} .
$$

The classical result on uniqueness of weak solutions in the class $L^{s}\left((0, T) ; L^{\gamma}\right)$ was given by Foias, Serrin and Masuda [1], [10], [9].

Theorem 2 (Foias-Serrin-Masuda). Let $a \in L_{\sigma}^{2}\left(\mathbb{R}^{d}\right)$. Let $u$ and $v$ are two weak solutions of (1.1) on $(0, T)$. Suppose that $u$ satisfies

$$
u \in L^{s}\left((0, T) ; L^{\gamma}\right) \quad \text { for } \quad \frac{2}{s}+\frac{d}{\gamma}=1 \quad \text { with } \quad d<\gamma<\infty .
$$

Assume that $v$ fulfills the energy inequality (2.2) for $0 \leq t<T$. Then we have $u=v$ on $[0, T)$.

REMARK 2. In Theorem 2, $v$ not need belong to the class (2.3). On the other hand, every weak solution $u$ with (2.3) fulfills the energy identity

$$
\|u(t)\|_{L^{2}}^{2}+2 \int_{0}^{t}\|\nabla u(s)\|_{L^{2}}^{2} d s=\|a\|_{L^{2}}^{2}, \quad 0 \leq t \leq T .
$$

It seems to be an interesting question whether every weak solution satisfies the energy inequality (2.2).

REMARK 3. The class (2.3) is important from the view point of scaling invariance for the Navier-Stokes equations. It can be easily seen that if is a pair of the solution to (1.1) on $\mathbb{R}^{d} \times(0, T)$, then so is the family $\left\{u_{\lambda}, p_{\lambda}\right\}_{\lambda>0}$ where

$$
u_{\lambda}(x, t)=\lambda u\left(\lambda x, \lambda^{2} t\right), \quad p_{\lambda}(x, t)=\lambda^{2} u\left(\lambda x, \lambda^{2} t\right) .
$$

Scaling invariance means that there holds

$$
\left\|u_{\lambda}\right\|_{L^{s}\left((0, \infty) ; L^{\gamma}\right)}=\left(\lambda^{1-\left(\frac{2}{s}+\frac{d}{\gamma}\right)}\|u\|_{L^{s}\left((0, \infty) ; L^{\gamma}\right)}\right)=\|u\|_{L^{s}\left((0, \infty) ; L^{\gamma}\right)} \quad \text { for all } \lambda>0
$$

if and only if

$$
\frac{2}{s}+\frac{d}{\gamma}=1
$$

We shall next deal with the critical case with $s=\infty$ and $\gamma=d$ in (2.3).

Theorem 3 (Masuda [9], Kozono-Sohr [5]). Let $a \in L_{\sigma}^{2}\left(\mathbb{R}^{d}\right)$. Let $u$ and $v$ be two weak solutions of (1.1) on $(0, T)$. Suppose that

$$
u \in L^{\infty}\left((0, T) ; L^{d}\right)
$$

and that $v$ fulfills the energy inequality (2.2) for all $0 \leq t<T$. Then we have $u=v$ on $[0, T)$.

REMARK 4. Masuda $[\mathbf{9}]$ proved that if $u \in L^{\infty}\left((0, T) ; L^{d}\right)$ is continuous from the right on $[0, T)$ in the norm of $L^{d}$, then there holds $u=v$ on $[0, T)$. Later on, Kozono-Sohr [5] showed that every weak solution in $L^{\infty}\left((0, T) ; L^{d}\right)$ of $(1.1)$ on $(0, T)$ becomes necessarily continuous from the right in the norm of $L^{d}$. 
The same result holds when, for $\gamma=+\infty$, we replace the assumption

$$
u \in L^{2}\left((0, T) ; L^{\infty}\right)
$$

by the weaker assumption

$$
\nabla u \in L^{2}\left((0, T) ; \dot{X}_{1}\left(\mathbb{R}^{d}\right)^{d}\right)
$$

The replacement of hypothesis $u \in L^{2}\left((0, T) ; L^{\infty}\right)$ by $\nabla u \in L^{2}\left((0, T) ; \dot{X}_{1}\left(\mathbb{R}^{d}\right)^{d}\right)$ was recently discussed in a similar context by Gala $[\mathbf{2}]$. Moreover, we have

TheOrem 4 (Gala). Let $a \in L_{\sigma}^{2}\left(\mathbb{R}^{d}\right)$ and let $u$, $v$ be two weak solutions of (1.1) on $(0, T)$. Suppose that

$$
\nabla u \in L^{2}\left((0, T) ; \dot{X}_{1}\left(\mathbb{R}^{d}\right)^{d}\right)
$$

and that $v$ fulfills the energy inequality (2.2) for $0 \leq t<T$. Then we have $u=v$ on $[0, T]$.

REMARK 5. By Theorem 2, every weak solution in $L^{2}\left((0, T) ; L^{\infty}\right)$ is unique.

Our result on uniqueness of the weak solution now reads :

THEOREM 5. Let $a \in L^{2}\left(\mathbb{R}^{d}\right)^{d}$ with $\nabla . a=0$ Assume that there exists a solution $u$ for the Navier-Stokes equations on $(0, T) \times \mathbb{R}^{d}$ (for some $T \in(0,+\infty]$ with some initial data a so that

$$
u \in L^{\infty}\left((0, T) ; L_{\sigma}^{2}\left(\mathbb{R}^{d}\right)^{d}\right) \cap L^{2}\left((0, T) ; \dot{H}_{\sigma}^{1}\left(\mathbb{R}^{d}\right)^{d}\right)
$$

and

$$
\nabla u \in L^{\frac{2}{2-r}}\left((0, T) ; \dot{X}_{r}\left(\mathbb{R}^{d}\right)^{d}\right) \quad \text { for all } 0 \leq r \leq 1 .
$$

Then, $u$ is the unique Leray-Hopf solution associated with a on $[0, T)$.

The following corollary, which is an immediate consequence of Theorem 5 gives a simpler sufficient condition in term of Lorentz spaces.

Corollary 1. Let $a \in L^{2}\left(\mathbb{R}^{d}\right)^{d}$ with $\nabla . a=0$ Assume that there exists a solution $u$ for the Navier-Stokes equations on $(0, T) \times \mathbb{R}^{d}$ (for some $T \in(0,+\infty]$ with some initial data a so that

$$
u \in L^{\infty}\left((0, T) ; L_{\sigma}^{2}\left(\mathbb{R}^{d}\right)^{d}\right) \cap L^{2}\left((0, T) ; \dot{H}_{\sigma}^{1}\left(\mathbb{R}^{d}\right)^{d}\right),
$$

and

$$
\nabla u \in L^{\frac{2}{2-r}}\left((0, T) ; L^{\frac{d}{r}, \infty}\left(\mathbb{R}^{d}\right)^{d}\right),
$$

where $L^{p, \infty}$ denotes the usual Lorentz (weak $L^{p}$ ) space. Then, $u$ is the unique Leray-Hopf solution associated with a on $[0, T)$.

The same result again holds when the assumption

$$
\nabla u \in L^{\frac{2}{2-r}}\left((0, T) ; L^{\frac{d}{r}, \infty}\left(\mathbb{R}^{d}\right)^{d}\right)
$$

is replaced by

$$
u \in L^{\frac{2}{2-r}}\left((0, T) ; L^{\frac{d}{r}}\left(\mathbb{R}^{d}\right)^{d}\right)
$$

We are now in a position to proof the main result. 
Proof. Let $v$ be another weak solution of (1.1) associated to $a$ on $(0, T)$ (with associated pressure $p$ ) such that

$$
v \in L^{\infty}\left((0, T) ; L_{\sigma}^{2}\left(\mathbb{R}^{d}\right)^{d}\right) \cap L^{2}\left((0, T) ; \dot{H}_{\sigma}^{1}\left(\mathbb{R}^{d}\right)^{d}\right)
$$

and

$$
\nabla u \in L^{\frac{2}{2-r}}\left((0, T) ; \dot{X}_{r}\left(\mathbb{R}^{d}\right)^{d}\right)
$$

We consider the difference $w=u-v$ and we obtain

$$
\begin{gathered}
\|u(t)\|_{L^{2}}^{2}+2 \int_{0}^{t}\|\nabla u(s)\|_{L^{2}}^{2} d s \leq\|a\|_{L^{2}}^{2}, \\
\|v(t)\|_{L^{2}}^{2}+2 \int_{0}^{t}\|\nabla v(s)\|_{L^{2}}^{2} d s \leq\|a\|_{L^{2}}^{2} .
\end{gathered}
$$

On the other hand, we have

$$
\langle u(t), v(t)\rangle+2 \int_{0}^{t}\langle\nabla u(s), \nabla v(s)\rangle d s=\|a\|_{L^{2}}^{2}+\int_{0}^{t}\langle w . \nabla u, w\rangle(s) d s
$$

for all $0 \leq t<T$. Combining the above inequalities, we obtain

$$
\begin{aligned}
& \|w(t)\|_{L^{2}}^{2}+2 \int_{0}^{t}\|\nabla w(s)\|_{L^{2}}^{2} d s \\
= & \|u(t)\|_{L^{2}}^{2}+2 \int_{0}^{t}\|\nabla u(s)\|_{L^{2}}^{2} d s+\|v(t)\|_{L^{2}}^{2} \\
& +2 \int_{0}^{t}\|\nabla v(s)\|_{L^{2}}^{2} d s-2\langle u(t), v(t)\rangle-4 \int_{0}^{t}\langle\nabla u(s), \nabla v(s)\rangle d s \\
\leq & -2 \int_{0}^{t}\langle w \cdot \nabla u, w\rangle d s .
\end{aligned}
$$

We thus observe that by Young inequality

$$
\left(a^{\alpha} b^{1-\alpha} \leq \alpha a+(1-\alpha) b \leq a+b \text { with } a, b \geq 0 \text { and } 0 \leq \alpha \leq 1\right),
$$

it follows that

$$
\begin{aligned}
\left|\int_{0}^{t}\langle w \cdot \nabla u, w\rangle\right| d s & \leq \int_{0}^{t}\|w \cdot \nabla u(s)\|_{L^{2}}\|w(s)\|_{L^{2}} d s \\
& \leq \int_{0}^{t}\|w(s)\|_{\dot{H}^{r}}\|\nabla u(s)\|_{\dot{X}_{r}}\|w(s)\|_{L^{2}} d s \\
& \leq \int_{0}^{t}\|w(s)\|_{L^{2}}^{1-r}\|\nabla w(s)\|_{L^{2}}^{r}\|\nabla u\|_{\dot{X}_{r}}\|w(s)\|_{L^{2}} d s \\
& \leq \int_{0}^{t}\left(\|w(s)\|_{L^{2}}^{2}\|\nabla u\|_{\dot{X}_{r}}^{\frac{2}{2-r}}\right)^{\frac{2-r}{2}}\left(\|\nabla w(s)\|_{L^{2}}^{2}\right)^{\frac{r}{2}} d s \\
& \leq \frac{1}{2} \int_{0}^{t}\|\nabla w\|_{L^{2}\left(\mathbb{R}^{d}\right)}^{2} d s+\frac{C}{2} \int_{0}^{t}\|w\|_{L^{2}\left(\mathbb{R}^{d}\right)^{d}}^{2}\|\nabla u\|_{\dot{X}_{r}\left(\mathbb{R}^{d}\right)}^{\frac{2}{2-r}} d s .
\end{aligned}
$$

where we used the following ones $(0 \leq r \leq 1)$

$$
\|\omega\|_{\dot{H}^{r}}=\frac{1}{(2 \pi)^{\frac{d}{2}}}\left\||\xi|^{r} \widehat{\omega}\right\|_{L^{2}} \leq\|\omega\|_{L^{2}}^{1-r}\|\nabla \omega\|_{L^{2}}^{r} .
$$


Hence by (2.7) there holds

$$
\|w(t)\|_{L^{2}}^{2}+\int_{0}^{t}\|\nabla w\|_{L^{2}}^{2} d \tau \leq C \int_{0}^{t}\|w\|_{L^{2}\left(\mathbb{R}^{d}\right)^{d}}^{2}\|\nabla v\|_{\dot{X}_{r}\left(\mathbb{R}^{d}\right)}^{\frac{2}{2-r}} d \tau
$$

for all $t>0$. Since $\nabla u \in L^{\frac{2}{2-r}}\left((0, T) ; \dot{X}_{r}\left(\mathbb{R}^{d}\right)^{d}\right)$ and since $w(0)=0$, it follows from the Gronwall inequality that

$$
\|w(t)\|_{L^{2}}^{2} \leq\|w(0)\|_{L^{2}}^{2} \exp \left(C \int_{0}^{t}\|\nabla u\|_{\dot{X}_{r}\left(\mathbb{R}^{d}\right)}^{\frac{2}{2-r}} d s\right),
$$

and thus

$$
\|w(t)\|_{L^{2}}^{2}=0, \quad 0 \leq t<T
$$

and implies uniqueness of weak solutions.

\section{References}

[1] Foias, C., Une remarque sur l' unicité des solutions des équations de Navier-Stokes endimension $n$, Bull. Soc. Math. France 89 (1961), 1-8.

[2] Gala, S., Remark on uniqueness of weak solutions to the Navier-Stokes equations, Analysis 28 (2008), 29-50.

[3] Hopf, E., Über die Anfangswertaufgabe für die hydrodynamishen Grundgleichungen, Math. Nach. 4 (1950/1951), 213-231.

[4] Kato, T., Strong $L^{p}$ solutions of the Navier-Stokes equations in Morrey spaces, Bol. Soc. Bras. Mat. 22,2 (1992) 127-155.

[5] Kozono, H., and Sohr, H., Remark on uniqueness of weak solutions to the Navier-Stokes equations, Analysis, 16 (1996), 255-271.

[6] Lemarié-Rieusset, P.G., Recent developments in the Navier-Stokes problem. Chapman \& Hall/ CRC Press, Boca Raton, 2002.

[7] Lemarié-Rieusset, P.G., and Gala.S., Multipliers between Sobolev spaces and fractional differentiation, J. Maths. Anal. Appl., 322 (2006), 1030-1054.

[8] Leray, J., Sur le mouvement d' un liquide visqueux emplissant l'espace. Acta. Math. 63 (1934), 193-248.

[9] Masuda, K., Weak solutions of Navier-Stokes equations, Tohoku Math J., 36 (1984), 623646.

[10] Serrin, J., On the interior regularity of weak solutions of the Navier stokes equations, Arch. Rational Mech. Anal. 9 (1962), 187 - 195.

[11] Stein, E. M. and Weiss, G., Introduction to Fourier Analysison euclidian spaces. Princeton Mathematical series. Princeton University Press, 1971.

University of Mostaganem, Department of Mathematics, B.P. 227, Mostaganem. Algeria

E-mail address: sadek.gala@gmail.com 